\title{
Transcranial direct current stimulation (tDCS) in addition to walking training on walking, mobility, and reduction of falls in Parkinson's disease: study protocol for a randomized clinical trial
}

Lucas Rodrigues Nascimento ${ }^{1,2}$, Ester Miyuki Nakamura-Palacios³, Augusto Boening ${ }^{1}$, Bárbara Naeme Lima Cordeiro ${ }^{1}$, Daniel Lyrio Cabral ${ }^{1}$, Alessandra Swarowsky ${ }^{4,5}$, Guilherme Peixoto Tinoco Arêas ${ }^{6}$, Wellingson Silva Paiva ${ }^{7}$ and Fernando Zanela da Silva Arêas ${ }^{1,3^{*}}$ (i)

\begin{abstract}
Background: Transcranial direct current stimulation (tDCS) has the potential to modulate cortical excitability and enhance the effects of walking training in people with Parkinson's disease. This study will examine the efficacy of the addition of tDCS to a task-specific walking training to improve walking and mobility and to reduce falls in people with Parkinson's disease.
\end{abstract}

Methods: This is a two-arm, prospectively registered, randomized trial with concealed allocation, blinded assessors, participants and therapists, and intention-to-treat analysis. Twenty-four individuals with Parkinson's disease, categorized as slow or intermediate walkers (walking speeds $\leq 1.0 \mathrm{~m} / \mathrm{s}$ ), will be recruited. The experimental group will undertake a 30-min walking training associated with tDCS, for 4 weeks. The control group will undertake the same walking training, but with sham-tDCS. The primary outcome will be comfortable walking speed. Secondary outcomes will include walking step length, walking cadence, walking confidence, mobility, freezing of gait, fear of falling, and falls. Outcomes will be collected by a researcher blinded to group allocation at baseline (week 0), after intervention (week 4), and 1 month beyond intervention (week 8).

Discussion: tDCS associated with walking training may help improve walking of slow and intermediate walkers with Parkinson's disease. If walking is enhanced, the benefits may be accompanied by better mobility and reduced fear of falling, and individuals may experience greater free-living physical activity at home and in the community.

Trial registration: Brazilian Registry of Clinical Trials (ReBEC) RBR-6bvnx6. Registered on September 23, 2019

Keywords: Clinical trial, Parkinson, Gait, Transcranial direct current stimulation, Rehabilitation

\footnotetext{
* Correspondence: fernandozanela@hotmail.com

${ }^{1}$ Center of Health Sciences, Discipline of Physical Therapy, Universidade

Federal do Espírito Santo (UFES), 1468 Marechal Campos Avenue, Maruípe,

Vitória, ES 29043900, Brazil

${ }^{3}$ Laboratory of Cognitive Sciences and Neuropsychopharmacology,

Department of Physiological Sciences, Universidade Federal do Espírito Santo

(UFES), Vitória, ES, Brazil

Full list of author information is available at the end of the article
}

\section{$\triangle B M C$}

(C) The Author(s). 2021, corrected publication 2021. Open Access This article is licensed under a Creative Commons Attribution 4.0 International License, which permits use, sharing, adaptation, distribution and reproduction in any medium or format, as long as you give appropriate credit to the original author(s) and the source, provide a link to the Creative Commons licence, and indicate if changes were made. The images or other third party material in this article are included in the article's Creative Commons licence, unless indicated otherwise in a credit line to the material. If material is not included in the article's Creative Commons licence and your intended use is not permitted by statutory regulation or exceeds the permitted use, you will need to obtain permission directly from the copyright holder. To view a copy of this licence, visit http://creativecommons.org/ licenses/by/4.0/. The Creative Commons Public Domain Dedication waiver (http://creativecommons.org/publicdomain/zero/1. 0/) applies to the data made available in this article, unless otherwise stated in a credit line to the data. 


\section{Background}

Parkinson's disease is the most common movement disorder and represents the second most common degenerative disease of the central nervous system, resulting from the death of dopamine-producing cells in the $s u b$ stantia nigra [1]. Disabilities associated with Parkinson's disease such as bradykinesia, impaired balance, and walking limitations can be present at initial diagnosis and progress over time $[1,2]$. In individuals with Parkinson's disease, while walking speed, step length, and mobility are typically reduced, fear of falling and the number of falls are increased [3, 4]. If walking performance is poor, community activity may be limited, and people may become housebound and isolated from the society. In addition, falls are a major determinant of poor quality of life, reduced mobility, and reduced life expectancy in people affected by Parkinson's disease [4].

Exercise and walking training have proved to be effective for improving walking and reducing falls early in people with Parkinson's disease [5-7]. Non-invasive brain stimulation by transcranial direct current stimulation (tDCS), which modulates cortical excitability by applying a direct current to the skull [8], could be associated with walking training and has the potential to enhance its benefits [9]. tDCS can increase the activity of the ventroposterolateral thalamic nucleus and may influence basal ganglia function. In addition, anodal tDCS over the motor cortex alter resting membrane potentials of underlying neurons leading to an increase in cortical excitability, with immediate and long-term effects that have been proposed to help improving motor skills $[8,10,11]$.

A Cochrane review [8] examined the effects of tDCS in individuals with Parkinson's disease. Meta-analyses, based on two trials, suggested that tDCS improves motor skills, measured by the Unified Parkinson's Disease Rating Scale (UPDRS) - part III-(MD - 14\%; 95\% CI - 25 to -4 ), but has no effect on walking speed (SMD 0.5; $95 \%$ CI -0.2 to 1.2 ) compared with sham intervention. More recently, a systematic review [12] reported that tDCS provides no clinically important benefits over walking training on walking speed, step length, or cadence in people with Parkinson's disease. Given that most trials included individuals with mild impairments or did not investigate effects on mobility and falls, the purpose of this randomized trial is to examine the effects of the addition of tDCS to walking training on walking, mobility, and falls in individuals with moderate walking limitations due to Parkinson's disease. The specific research questions are as follows:

1. In people with Parkinson's disease, is walking training associated with tDCS superior to walking training alone for improving walking (speed, step length, cadence, confidence), mobility, and falls?
2. Are any benefits maintained beyond the intervention period?

\section{Methods \\ Design}

A prospective, randomized controlled trial with concealed allocation, blinded assessors, participants and therapists, and intention-to-treat analysis will be carried out (Fig. 1). Community-dwelling people with Parkinson's disease will be recruited from the general community, by means of advertisements and by screening public rehabilitation services and lists of previous observational or cross-sectional research projects. Participants will be randomly allocated into either experimental group (i.e., walking training with tDCS) or control group (i.e., walking training with sham-tDCS). Outcome measures will be collected by trained researchers at baseline (week 0), at the end of the intervention (week 4), and 1 month beyond the intervention (week 8). Measurements and interventions will be conducted during the on phase of medication. Analyses of the inclusion criteria, getting the informed consent, data collection, and statistical analyses will be carried out by researchers, who will be blind to the group allocation. All the participants will be evaluated and receive all the information regarding the interventions in a research laboratory. The study obtained ethical approval from the Institutional Research Ethical Committee (CAAE 06952819.6.0000.5060) of the Universidade Federal do Espírito Santo, Vitória, Brazil. The trial was prospectively registered at the Ensaiosclinicos. gov.br, Registry: RBR-6bvnx6 (www.ensaiosclinicos.gov. br/rg/RBR-6bvnx6/).

\section{Participants and therapists-inclusion and exclusion criteria}

Participants will be individuals with Parkinson's disease, who will be eligible if they:

- Are $>40$ years of age

- Are able to walk at least $14 \mathrm{~m}$, independently, with or without assistive devices

- Walk at speeds $\leq 1.0 \mathrm{~m} / \mathrm{s}$

- Have experienced a freezing episode over the past month, according to the part I of the New Freezing of Gait Questionnaire [13]

- Have adapted to their current anti-Parkinsonian medication for at least 2 weeks

- Provide written consent

They will be excluded if they:

- Have cognitive deficits, which will be screened by the Mini-Mental State Examination. The cutoff scores are 26 for people with high levels of 


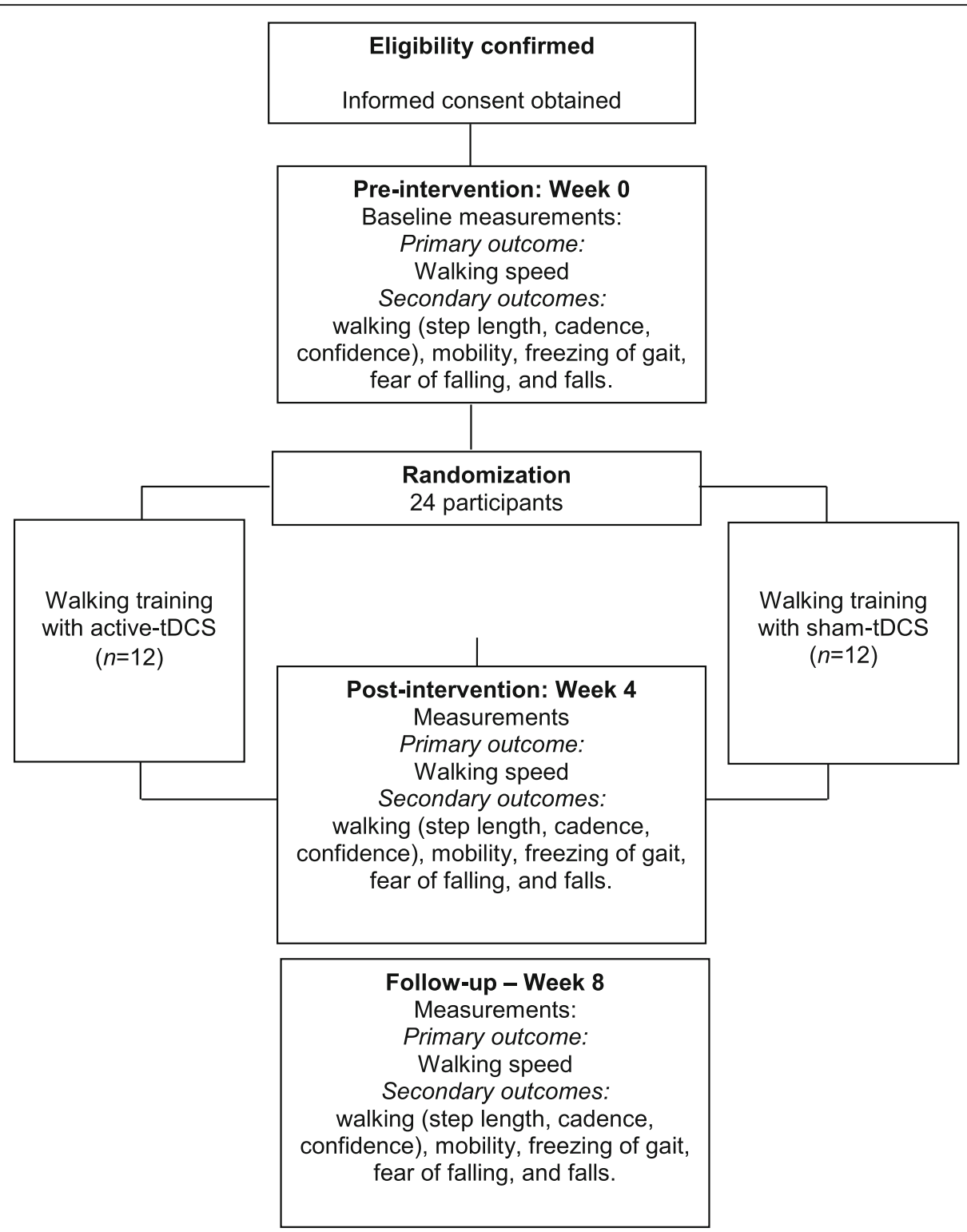

Fig. 1 Design of the trial

education, 18 for people with elementary and middle levels, and 13 for illiterate people [14].

- Suffer from unstable cardiovascular disease or other uncontrolled chronic conditions that would interfere with the safety and conduct of the training and testing protocol or interpretation of the results.

- Had undergone deep brain stimulation.

Therapists, who will deliver the intervention, will be eligible if they have received training from the research leaders (FZSA and LRN), who have more than 10 years of clinical experience in the area of neurological rehabilitation. A research assistant will be responsible for setting the stimulator on active or sham-tDCS, so therapists delivering the intervention will be blind to the group allocation.

\section{Randomization}

Randomization will be computer-generated, by a researcher not involved in participant recruitment, and stratified according to the baseline walking speeds: slow $(\leq 0.5 \mathrm{~m} / \mathrm{s})$ and intermediate $(0.51$ to $1.0 \mathrm{~m} / \mathrm{s})$ walkers, to ensure an even spread between the groups (1:1 allocation). The allocation of the participants will be concealed in sequentially numbered and sealed in opaque envelopes, prepared prior to the study by a research assistant, who will not be involved in the study. After the baseline measures have been collected, participants will be 
randomly assigned to the experimental or control group by the treating therapist, after revealing the content of the sealed opaque envelopes. All outcomes will be measured by blinded assessors. All enrolled participants will receive a code, in order to protect confidentiality before, during, and after the trial.

\section{Intervention}

The experimental group will undertake a task-specific walking training associated with tDCS, 30 min per day, 3 days per week, over 4 weeks, i.e., 12 sessions of tDCS. A stimulator (DC-Stimulator Plus, NeuroConn, Ilmenau, Germany, or Neuroeletrics STARTIM tCS, Barcelona, Spain) will deliver a continuous direct current by a saline-soaked pair of surface sponge electrodes (size of electrodes $35 \mathrm{~cm}^{2}$ ). The anode will be placed at the $\mathrm{Cz}$ position on the scalp, corresponding to the location of the supplementary motor area, in accordance with the International EEG 10/20 system [15, 16]. The cathode will be positioned over the left supraorbital area. Participants will receive electrical stimulation of $2 \mathrm{~mA}$, during the walking training. The $30-\mathrm{min}$ sessions of taskspecific walking training will include the practicing part of the task (about $10 \mathrm{~min}$ ), where the muscles are working in a manner similar to a full task performance and practicing the whole task (about $20 \mathrm{~min}$ ) [17]. Table 1 shows the ten walking activities of the task-specific walking training, which will be individually tailored for each participant.

The control group will undertake the task-specific walking training associated with a sham-tDCS. The control group will receive the same walking training, electrode positioning, and testing schedule as the experimental group. This will avoid bias related to the type and amount of attention given to the participants. If the addition of $\mathrm{tDCS}$ proves to be effective, the control group may receive the experimental training program after the experiment is complete.

The intervention will be undertaken in Clinics of Physiotherapy at the Universidade Federal do Espírito Santo. To encourage the participants to comply with the protocol, both groups will be asked to sign a symbolic contract of commitment to the proposed protocol. Participants will not be informed whether they are receiving tDCS or sham-tDCS.

\section{Primary outcome}

The primary outcome is comfortable walking speed, measured by the $10-\mathrm{m}$ Walk Test, and reported in $\mathrm{m} / \mathrm{s}$. The participants will be instructed to walk at their "comfortable speed" along a 14-m hallway, and the time to cover the central $10 \mathrm{~m}$ will be recorded with a digital stopwatch and converted to speed [18]. After a practice trial, the value obtained during a single test will be used for analysis [19].

\section{Secondary outcomes}

Secondary outcomes are walking step length, walking cadence, walking confidence, mobility, freezing of gait, fear of falling, and falls.

Walking step length and cadence will be measured using the 10-m Walk Test. Step length will be calculated by dividing the covered distance, i.e., $10 \mathrm{~m}$, by the number of steps to cover the distance, and reported in meters. Walking cadence will be calculated by dividing the number of steps by the time to cover the distance, i.e., $10 \mathrm{~m}$, and reported in steps $/ \mathrm{min}$.

Walking confidence will be measured using the Brazilian version of the modified Gait Efficacy Scale and reported as scores ranging from 10 to 100 . This scale is a 10-item measure that addresses the perception of the level of confidence in walking during challenging

Table 1 Activities of the task-specific walking training

\begin{tabular}{|c|c|c|c|}
\hline Activity & Type & Practice & Progression \\
\hline Step on block & Segmented & Swing & $\begin{array}{l}\text { Diminishing hand support and increasing speed, height, and/or } \\
\text { distance }\end{array}$ \\
\hline $\begin{array}{l}\text { Stand on one leg then perform } \\
\text { plantarflexion }\end{array}$ & & Stance & Diminishing hand support and increasing speed \\
\hline Step sideways & & $\begin{array}{l}\text { Lateral } \\
\text { movement }\end{array}$ & Diminishing hand support and increasing speed and/or distance \\
\hline Step sideways on a block & & $\begin{array}{l}\text { Lateral } \\
\text { movement }\end{array}$ & $\begin{array}{l}\text { Diminishing hand support and increasing speed, height, and/or } \\
\text { distance }\end{array}$ \\
\hline Walk on footprints & Complete & Whole task & Increasing step length \\
\hline Walk with an auditory cue & & Whole task & Increasing cadence \\
\hline Walk and turn & & Whole task & Increasing speed \\
\hline Walk and cross obstacles & & Whole task & Increasing speed \\
\hline Dual-task walk & & Whole task & Increasing speed and cognitive/motor demands \\
\hline Overground and treadmill speed walk & & Whole task & Increasing speed \\
\hline
\end{tabular}


circumstances. The items include walking on a level surface and on grass, stepping over an obstacle, stepping up and down a curb, ascending and descending stairs (with and without a handrail), and walking over a long distance. The items are individually scored on a 10-point Likert scale, with 1 indicating "no confidence" and 10 indicating "complete confidence," [20, 21].

Mobility will be measured by the Timed-up and Go Test (TUG) and reported as seconds. The participants will be seated in a chair with their backs against the chair back. On the command "go," the participants will be instructed to rise from the chair, walk $3 \mathrm{~m}$ at a comfortable and safe pace, turn, walk back to the chair, and sit down. After a practice trial, the value obtained during a single test will be used for analysis [19, 22].

Freezing of gait will be measured using parts II and III of the New Freezing of Gait Questionnaire and reported as scores ranging from 0 to 28 , where higher scores indicate worse episodes of freezing. The New Freezing of Gait Questionnaire is a reliable tool to detect and evaluate the impact and severity of freezing of gait, when applied to patients (ICC $=0.88$ ) or caregivers (ICC $=0.97)$ [13].

Fear of falling will be measured using the Brazilian version of the Falls Efficacy Scale - International (FES-I Brazil) and reported as scores ranging from 16 to 64, where higher scores indicate greater fear of falling. The FES-I Brazil measures the fear of falling during 16 activities of daily living and has appropriate internal consistency $(\alpha=0.93)$ and intra- and inter-examiner reliability $(\mathrm{ICC}=0.84$ and ICC $=0.91)$ [23].

The number of falls will be recorded by the use of a "falls diary" [24], and the proportion of fallers in each group will also be compared. All participants will receive weekly calendars on entry to the study, with instructions to record the following events: number of falls, visits by or to nursing and allied health personnel, and hospitalizations. Participants will be asked to return the completed calendar weekly to a researcher unaware of the group allocation.

\section{Data monitoring body}

An independent researcher, who will be blind to the group allocations, will monitor any adverse effects and perform database management and statistical analyses. The treating therapists will be responsible for the monitoring of doses and compliance.

\section{Sample size estimation}

Twenty-four participants will be recruited, with walking speed as the primary outcome. The sample size has been calculated, to reliably detect a between-group difference of $0.18 \mathrm{~m} / \mathrm{s}$ in walking speed, with $80 \%$ power, at a twotailed significance level of 0.05 . In previous trials $[9,16$, 25 ] with similar samples of community-dwelling people with Parkinson's disease who received tDCS associated with walking training, the mean walking speed of the participants was $0.73 \mathrm{~m} / \mathrm{s}(\mathrm{SD} 0.15 \mathrm{~m} / \mathrm{s})$, measured by a timed-walking measure. The least number of participants needed to detect a $0.18 \mathrm{~m} / \mathrm{s}$ difference between two independent groups, which would indicate a change in the UPDRS walking category [26], is 11 per group, i.e., 22 participants in total. Based on the assumption that about $10 \%$ of participants may drop out during the study, a target of 24 participants in total has been set.

\section{Statistical analyses}

Data collection will yield eight variables: walking speed $(\mathrm{m} / \mathrm{s})$, walking step length $(\mathrm{m})$, walking cadence (steps/ min), walking confidence (modified Gait Efficacy Scale score; 10-100), mobility (TUG; seconds), freezing of gait (New Freezing of Gait Questionnaire; 0-28), fear of falling (FES-I Brazil score; 16-64), and number of falls. There are two factors (group $\times$ time), with repeated measures on the time factor. Two-way analyses of variance with repeated measures at all time points for all outcomes will be reported to evaluate the statistical significance of the between-group differences. The mean between-group differences, along with $95 \%$ confidence intervals, will be reported for all outcomes. The effect of the intervention will be calculated based on intentionto-treat analyses.

\section{Study organization and funding}

This trial will be conducted according to relevant ethical frameworks and has received approval from the institutional ethical review board. It is funded by the Brazilian National Funding Agency: Fundação de Amparo à Pesquisa e Inovação do Espírito Santo (FAPES). The results will be submitted for publication in journals related to the area of neurorehabilitation, and access to the final trial dataset may be obtained from the authors based upon reasonable request.

\section{Discussion}

This trial will examine the efficacy of the addition of tDCS to walking training for improving walking, mobility, and falls in people with Parkinson's disease. Although previous studies $[9,10,15,16,27]$ have investigated the combined effect of tDCS and walking training, methodological shortcomings (e.g., design and very small sample sizes), characteristics of interventions (e.g., duration of intervention, type of exercises), and characteristics of the participants (e.g., level of disabilities) prevent drawing clear conclusions, which could help clinicians in their decision-making process. In addition, many trials did not investigate whether benefits carry over to improving mobility and falls. In response to this challenge, a triple-blinded randomized trial will be 
conducted. High internal validity is expected, due to randomization, concealed allocation, blinding of assessors, participants and therapists, intention-to-treat analysis [28], and appropriate sample size.

Previous studies suggest that tDCS modulates cortical excitability during stimulation by non-synaptic changes of the cells, and increasing evidence indicates that the aftereffects of tDCS are driven by synaptic modification [29]. Synaptic plasticity could, therefore, induce longlasting excitability changes in the central nervous system. Although the neurophysiological effects of tDCS have been identified and its safety has been proved [29, 30], the clinical effects remain unclear. This trial focuses on identifying the effects of the addition of tDCS on walking parameters commonly affected in individuals with Parkinson's disease [3, 31]. If neurophysiological benefits of tDCS are carried over to clinical benefits, clinicians will assure an important tool to help reduce disabilities related to the continuous death of dopamine-producing cells in the substantia nigra. Less expensive portable devices are currently available, making direct current potentially ideal as an adjunct to other physical interventions [30].

This trial has some limitations. The experimental and control interventions consist of walking exercises delivered three times per week over 4 weeks and, therefore, depend on participants' motivation, adherence, and commitment. Strategies to encourage participants to comply with the protocol, such as contracts and phone calls, are planned.

In conclusion, the results of this trial may result in an important advance in neurological rehabilitation. First, an adjunct intervention may help improve walking of slow and intermediate walkers with Parkinson's disease. Second, if walking is enhanced, the benefits may be accompanied by better mobility and reduced fear of falling. Individuals may experience greater free-living physical activity at home and in the community, increased social interactions, and increased ability to engage in work and leisure activities [32, 33], which is the ultimate goal for both patients and rehabilitation professionals.

\section{Trial status}

Recruitment has started in September 2019 according to the registry RBR-6bvnx6, version 1.0, September 23, 2019. At the time of manuscript submission, the expected duration of the study, including enrollment and statistical analysis, should be 5 years. The approximate date of planned recruitment completion is December 31, 2023.

\section{Abbreviations}

tDCS: Transcranial direct current stimulation; UPDRS: Unified Parkinson's Disease Rating Scale; TUG: Timed-up and Go Test; FES-I: Falls Efficacy Scale International; steps/min: Steps per minute
Acknowledgements

None declared.

\section{Ancillary and post-trial care}

Patients that are enrolled in the study may receive additional medical or rehabilitation procedures to cover for harms associated with the protocol at the university hospital or rehabilitation clinics at the Universidade Federal do Espírito Santo.

\section{Dissemination plans}

Preliminary results of the trial may be submitted as conference abstracts for specialized discussion regarding the main findings. The final report will be submitted for publication in peer-reviewed journals related to neuroscience and neurorehabilitation. In addition, the included participants will receive an easy-to-read report explaining the research findings. The communications aimed at a broader audience will be in accordance with the Checklist for Communicating Science and Health Research to the Public, developed by the National Institutes of Health (www.nih.gov).

\section{Authors' contributions}

All authors contributed to the development of the study protocol and this manuscript. All authors have read and approved the final version of the manuscript.

\section{Funding}

This work was supported by the Fundação de Amparo à Pesquisa e Inovação do Espírito Santo - FAPES (Universal - 021/2018), Brazil.

\section{Availability of data and materials}

The study investigators have full access to the study datasets. The datasets used and analyzed during the study are available from the corresponding author on reasonable request; however, any information shared will be blinded to any identifying participant information. The trial results will be communicated to healthcare professionals and other relevant groups via publications, reporting in results databases, and presenting the data during the medical congresses and conferences.

\section{Declarations}

Ethics approval and consent to participate

The study obtained ethical approval from the Institutional Research Ethical Committee (CAAE 06952819.6.0000.5060) of the Universidade Federal do Espírito Santo, Vitória, Brazil, which means all of the study procedures remain in accordance with the Declaration of Helsinki concerning the Ethical Principles for Medical Research Involving Human Subjects. Informed consent will be obtained from all study participants.

Consent for publication

Not applicable.

\section{Competing interests}

The authors declare that they have no competing interests.

\section{Author details}

${ }^{1}$ Center of Health Sciences, Discipline of Physical Therapy, Universidade Federal do Espírito Santo (UFES), 1468 Marechal Campos Avenue, Maruípe, Vitória, ES 29043900, Brazil. ${ }^{2}$ NeuroGroup, Department of Physical Therapy, Universidade Federal de Minas Gerais (UFMG), Belo Horizonte, MG, Brazil. ${ }^{3}$ Laboratory of Cognitive Sciences and Neuropsychopharmacology, Department of Physiological Sciences, Universidade Federal do Espírito Santo (UFES), Vitória, ES, Brazil. ${ }^{4}$ Department of Physical Therapy, Universidade Estadual de Santa Catarina (UDESC), Florianópolis, SC, Brazil. ${ }^{5}$ Doctor of Physical Therapy Program, Advent Health University, Orlando, USA. ${ }^{6}$ Department of Human Physiology, Universidade Federal do Amazonas (UFAM), Manaus, AM, Brazil. ${ }^{7}$ Neurosurgery Division, Department of Neurology, Clinical Hospital, Faculty of Medicine, University of São Paulo, São Paulo, Brazil. 
Received: 1 December 2020 Accepted: 5 September 2021

Published online: 21 September 2021

\section{References}

1. Tysnes OB, Storstein A. Epidemiology of Parkinson's disease. J Neural Transm (Vienna). 2017;124(8):901-5. https://doi.org/10.1007/s00702-017-1686-y.

2. Kim SD, Allen NE, Canning CG, Fung VSC. Chapter 11 - Parkinson disease. Handb Clin Neurol. 2018;159:173-93. https://doi.org/10.1016/B978-0-444-63 916-5.00011-2.

3. Moon Y, Sung J, An R, Hernandez ME, Sosnoff JJ. Gait variability in people with neurological disorders: a systematic review and meta-analysis. Hum Mov Sci. 2016;47:197-208. https://doi.org/10.1016/j.humov.2016.03.010.

4. Fasano A, Canning CG, Hausdorff JM, Lord S, Rochester L. Falls in Parkinson's disease: a complex and evolving picture. Mov Disord. 2017;32(11):1524-36. https://doi.org/10.1002/mds.27195.

5. Mehrholz J, Kugler J, Storch A, Pohl M, Elsner B, Hirsch K. Treadmill training for patients with Parkinson's disease. Cochrane Database Syst Rev. 2015;9: CD007830. https://doi.org/10.1002/14651858.CD007830.pub3.

6. Canning CG, Sherrington C, Lord SR, Close JCT, Heritier S, Heller GZ, et al. Exercise for falls prevention in Parkinson disease: a randomized controlled trial. Neurology. 2015;84(3):304-12. https://doi.org/10.1212/WNL. 0000000000001155 .

7. Spaulding SJ, Barber B, Colby M, Cormack B, Mick T, Jenkins ME. Cueing and gait improvement among people with Parkinson's disease: a meta-analysis. Arch Phys Med Rehabil. 2013;94(3):562-70. https://doi.org/10.1016/j.apmr.2 012.10.026.

8. Elsner B, Kugler J, Pohl M, Mehrholz J. Transcranial direct current stimulation (tDCS) for idiopathic Parkinson's disease. Cochrane Database Syst Rev. 2016; 7:CD010916. https://doi.org/10.1002/14651858.CD010916.pub2.

9. Kaski D, Dominguez RO, Allum JH, Islam AF, Bronstein AM. Combining physical training with transcranial direct current stimulation to improve gait in Parkinson's disease: a pilot randomized controlled study. Clin Rehabil. 2014:28(11):1115-24. https://doi.org/10.1177/0269215514534277.

10. Schabrun SM, Lamont RM, Brauer SG. Transcranial direct current stimulation to enhance dual-task gait training in Parkinson's disease: a pilot RCT. PLoS One. 2016;11(6):e0158497. https://doi.org/10.1371/journal.pone.0158497.

11. Nitsche MA, Paulus W. Sustained excitability elevations induced by transcranial DC motor cortex stimulation in humans. Neurology. 2001;57(10): 1899-1. https://doi.org/10.1212/WNL.57.10.1899.

12. Nascimento LR. do Carmo WA, de Oliveira GP, Arêas FZDS, Dias FMV. Transcranial direct current stimulation provides no clinically important benefits over walking training for improving walking in Parkinson's disease: a systematic review. J Physiother. 2021;67(3):190-6. https://doi.org/10.1016/j. jphys.2021.06.003.

13. Nieuwboer A, Rochester $L$, Heman $T$, et al. Reliability of the new freezing og gait questionnaire: agreement between patients with Parkinson's disease and their carers. Gait Posture. 2009;30(4):459-63. https://doi.org/10.1016/j.ga itpost.2009.07.108.

14. Bertolucci P, Brucki S, Campacci S, Juliano Y. The Mini-Mental State Examination in an outpatient population: influence of literacy. Arq Neuropsiquiatr. 1994;52(1):1-7. https://doi.org/10.1590/S0004-282X19940001 00001.

15. Klem GH, Lüders HO, Jasper HH, Elger C. The ten-twenty electrode system of the International Federation of Clinical Neurophysiology. Electroencephalogr Clin Neurophysiol Suppl. 1999;52:3-6 PMID: 10590970

16. Yotnuengnit $P$, Bhidayasiri $R$, Donkhan $R$, Chaluaysrimuang J, Piravej $K$. Effects of transcranial direct current stimulation plus physical therapy on gait in patients with Parkinson disease: a randomized controlled trial. Am J Phys Med Rehabil. 2018;97(1):7-15. https://doi.org/10.1097/PHM. 0000000000000783.

17. Scianni A, Teixeira-Salmela LF, Ada L. Effect of strengthening exercise in addition to task-specific gait training after stroke: a randomised trial. Int J Stroke. 2010;5(4):329-35. https://doi.org/10.1111/j.1747-4949.2010.00449.x.

18. Nascimento LR, Caetano LC, Freitas DC, Morais TM, Polese JC, TeixeiraSalmela LF. Different instructions during the ten-meter walking test determined significant increases in maximum gait speed in individuals with chronic hemiparesis. Braz J Phys Ther. 2012;16(2):122-7. https://doi.org/10.1 590/S1413-35552012005000008.

19. Faria CD, Teixeira-Salmela LF, Neto MG, Rodrigues-de-Paula F. Performancebased tests in subjects with stroke: outcome scores, reliability and measurement errors. Clin Rehabil. 2011;26(5):460-9. https://doi.org/10.1177/ 0269215511423849 .

20. Newell AM, VanSwearingen JM, Hile E, Brach JS. The modified Gait Efficacy Scale: establishing the psychometric properties in older adults. Phys Ther. 2012;92(2):318-28. https://doi.org/10.2522/ptj.20110053.

21. Avelino PR, Menezes KKP, Nascimento LR, et al. Cross-cultural adaptation of the Modified Gait Efficacy Scale for individuals with stroke. Rev Ter Ocup Univ São Paulo. 2018;29(3):230-6. https://doi.org/10.11606/issn.2238-6149.v2 9i3p230-236.

22. Podsiadlo D, Richardson S. The timed "up \& go": a test of basic functional mobility for frail elderly persons. J Am Geriatr Soc. 1991;39(2):142-8. https:// doi.org/10.1111/j.1532-5415.1991.tb01616.x.

23. Camargos FFO, Dias RC, Dias JMD, Freire MTF. Cross-cultural adaptation and evaluation of the psychometric properties of the Falls Efficacy Scale International Among Elderly Brazilians (FES-I-BRAZIL). Braz J Phys Ther. 2010; 14(3):237-43. https://doi.org/10.1590/S1413-35552010000300010.

24. Canning CG, Sherrington C, Lord SR, Fung VSC, Close JCT, Latt MD, et al. Exercise therapy for prevention of falls in people with Parkinson's disease: a protocol for a randomised controlled trial and economic evaluation. BMC Neurol. 2009;9(1):4. https://doi.org/10.1186/1471-2377-9-4.

25. da Silva DCL, Lemos T, de Sá FA, et al. Effects of acute transcranial direct current stimulation on gait kinematics of individuals with Parkinson disease. Topics Geriatr Rehabil. 2018;34(4):262-8. https://doi.org/10.1097/TGR. 0000000000000203.

26. Hass CJ, Bishop M, Moscovich M, Stegemöller EL, Skinner J, Malaty IA, et al. Defining the clinically meaningful difference in gait speed in persons with Parkinson disease. J Neurol Phys Ther. 2014;38(4):233-8. https://doi.org/10.1 097/NPT.0000000000000055.

27. Costa-Ribeiro A, Maux A, Bosford T, Aoki Y, Castro R, Baltar A, et al. Transcranial direct current stimulation associated with gait training in Parkinson's disease: a pilot randomized clinical trial. Dev Neurorehabil. 2017; 20(3):121-8 https://doi.org/10.3109/17518423.2015.1131755.

28. Slack MK, Draugalis JR. Establishing the internal and external validity of experimental studies. Am J Health Syst Pharm. 2001;58(22):2173-81. 11 760921. https://doi.org/10.1093/ajhp/58.22.2173.

29. Stagg CJ, Nitsche MA. Physiological basis of transcranial direct current stimulation. Neuroscientist. 2011;17(1):37-53. https://doi.org/10.1177/1073 858410386614.

30. Schabrun SM, Chipchase LS. Priming the brain to learn: the future of therapy? Man Ther. 2012;17(2):184-6. https://doi.org/10.1016/j.math.2011.12. 001.

31. Speciali DS, Oliveira EM, Cardoso JR, Correa JC, Baker R, Lucareli PR. Gait Profile Score and movement analysis profile in patients with Parkinson's disease during concurrent cognitive load. Braz J Phys Ther. 2014;18(4):31522. https://doi.org/10.1590/bjpt-rbf.2014.0049.

32. Zaman A, Ellingson L, Sunken A, Gibson E, Stegemöller EL. Determinants of exercise behaviour in persons with Parkinson's disease. Disabil Rehabil. 2019; 43(5):696-702. https://doi.org/10.1080/09638288.2019.1638975.

33. Hammarlund CS, Andersson $K$, Andersson M, Nilsson MH, Hagell P. The significance of walking from the perspective of people with Parkinson's disease. J Parkinsons Dis. 2014;4(4):657-63. https://doi.org/10.3233/JPD-140399.

\section{Publisher's Note}

Springer Nature remains neutral with regard to jurisdictional claims in published maps and institutional affiliations.

Ready to submit your research? Choose BMC and benefit from:

- fast, convenient online submission

- thorough peer review by experienced researchers in your field

- rapid publication on acceptance

- support for research data, including large and complex data types

- gold Open Access which fosters wider collaboration and increased citations

- maximum visibility for your research: over $100 \mathrm{M}$ website views per year

At $\mathrm{BMC}$, research is always in progress.

Learn more biomedcentral.com/submission 